\title{
The pleiotropic effects of metformin: time for prospective studies
}

\author{
Daniel I Bromage and Derek M Yellon ${ }^{*}$
}

\begin{abstract}
The global prevalence of diabetes has risen to epidemic proportions and the trend is predicted to continue. The consequent burden of cardiovascular morbidity and mortality is a major public health concern and new treatments are required to mitigate the deleterious effects of cardiovascular disease in diabetic patients. Ischaemia-reperfusion injury is well known to exacerbate the harmful effects of acute myocardial infarction and subsequent therapeutic reperfusion, and several mechanical and pharmacological approaches to mitigating this injury have been investigated. Metformin, which is cheap, relatively safe and widely used in type 2 diabetes, is one such pharmacotherapy with considerable pre-clinical evidence for cardioprotective utility beyond its glucose-lowering effect. However, despite convincing basic evidence its translation to clinical application has largely been limited to studies of cardiovascular risk. There are several barriers to prospective randomized assessment in the context of acute myocardial infarction, not least the accessibility and already widespread use of metformin among patients with type 2 diabetes at high risk of cardiovascular events. In the place of class 1 evidence, well-designed prospective cohort studies of the potential pleiotropic utility of metformin in cardiovascular disease, and particularly its benefit in ischaemia-reperfusion injury, are needed. Given the availability of metformin worldwide, this is particularly true in low- and middle-income countries where the optimal therapy for acute myocardial infarction, primary percutaneous coronary intervention, may not be available, and instead patients are managed with thrombolysis. As this is less effective, metformin as an adjunct to thrombolysis (or PPCl) could represent an effective, cheap means of cardioprotection with global relevance.
\end{abstract}

Keywords: Metformin, Cardioprotection, Ischaemia-reperfusion injury, STEMI, Type 2 diabetes, PCI, Thrombolysis

\section{Background}

The global prevalence of diabetes was estimated to be $9 \%$ in 2014 and this looks set to rise dramatically [1, 2]. Cardiovascular disease accounts for $52 \%$ of deaths among patients with type 2 diabetes [3], and therefore constitutes an important target for intervention. Of this excess cardiovascular mortality, an estimated $39 \%$ is attributable to ST-segment elevation myocardial infarction (STEMI) [4]. Following STEMI, early reperfusion by primary percutaneous coronary intervention (PPCI) is the most effective strategy for reducing infarct size and improving clinical outcome $[5,6]$. However, adverse sequelae persist: in a recent study, 30-day, 1-year, and 5-year all-cause (and cardiac) mortality rates following PPCI for STEMI were

*Correspondence: d.yellon@ucl.ac.uk

The Hatter Cardiovascular Institute, 67 Chenies Mews, London WC1E 6HX, UK
$7.9 \%$ (7.3 \%), $11.4 \%$ (8.4 \%), and $23.3 \%$ (13.8 \%), respectively [7]. It is known that patients with diabetes and coronary heart disease suffer worse clinical outcomes $[8,9]$, and in the aforementioned observational study, diabetes significantly increased the hazard of death over a median follow-up period of 4.7 years (fully adjusted HR 1.62 [95 \% CI 1.32-1.97], $\mathrm{p}<0.001$ ).

A potential target in STEMI patients is the paradoxical injury inflicted by the therapeutic restoration of blood flow, known as ischaemia-reperfusion injury (IRI), which may exacerbate the final infarct size [10-13]. Several mechanical and pharmacological interventions have been investigated with respect to their ability to attenuate IRI [14], but to date no agent is in routine clinical use to protect the myocardium against IRI. Is there a role for metformin in this regard? Metformin is an oral antidiabetic agent of the biguanide class that exerts its effect by suppressing gluconeogenesis and increasing peripheral 
glucose uptake. However, the translation of metformin from a promising cardioprotective agent in the laboratory setting (see below) to implementation at the bedside has stalled, perhaps as a result of the widespread uptake of metformin as the first-line oral hypoglycaemic among patients with type 2 diabetes in high-income countries. Nonetheless, metformin is inexpensive and relatively widely available and its repurposing in STEMI could confer morbidity and mortality benefits both in the context of PPCI and in regions where PPCI is not readily available. To test this hypothesis, well-designed prospective cohort studies of the role of metformin in IRI are required.

\section{Basic evidence}

Several groups have demonstrated a significant reduction in infarct size in animal models of IRI following the administration of metformin [15-18]. It is hypothesized that the cardioprotective effect of metformin against IRI is independent of its hypoglycaemic actions [18]. We and others have investigated potential mechanisms for this phenomenon, which are thought to include activation of the RISK pathway either directly [15], by increased AMPK activation [17, 19], or via adenosine receptor stimulation [16], all of which inhibit mPTP opening at reperfusion and effect cardioprotection (see Fig. 1) [20, 21]. Interestingly, metformin has also been shown to reduce myocardial infarct size when administered $24 \mathrm{~h}$ and chronically prior to index ischaemia $[18,22]$.

\section{Clinical evidence}

The UK Prospective Diabetes Study (UKPDS) was a large randomised, multicentre trial of glycaemic therapies in 5,102 patients with newly diagnosed type 2 diabetes [8]. It reported that metformin reduced the risk of AMI when compared to diet therapy alone in diabetic patients. Furthermore, metformin reduced all-cause and cardiovascular mortality compared to sulfonylureas and insulin,

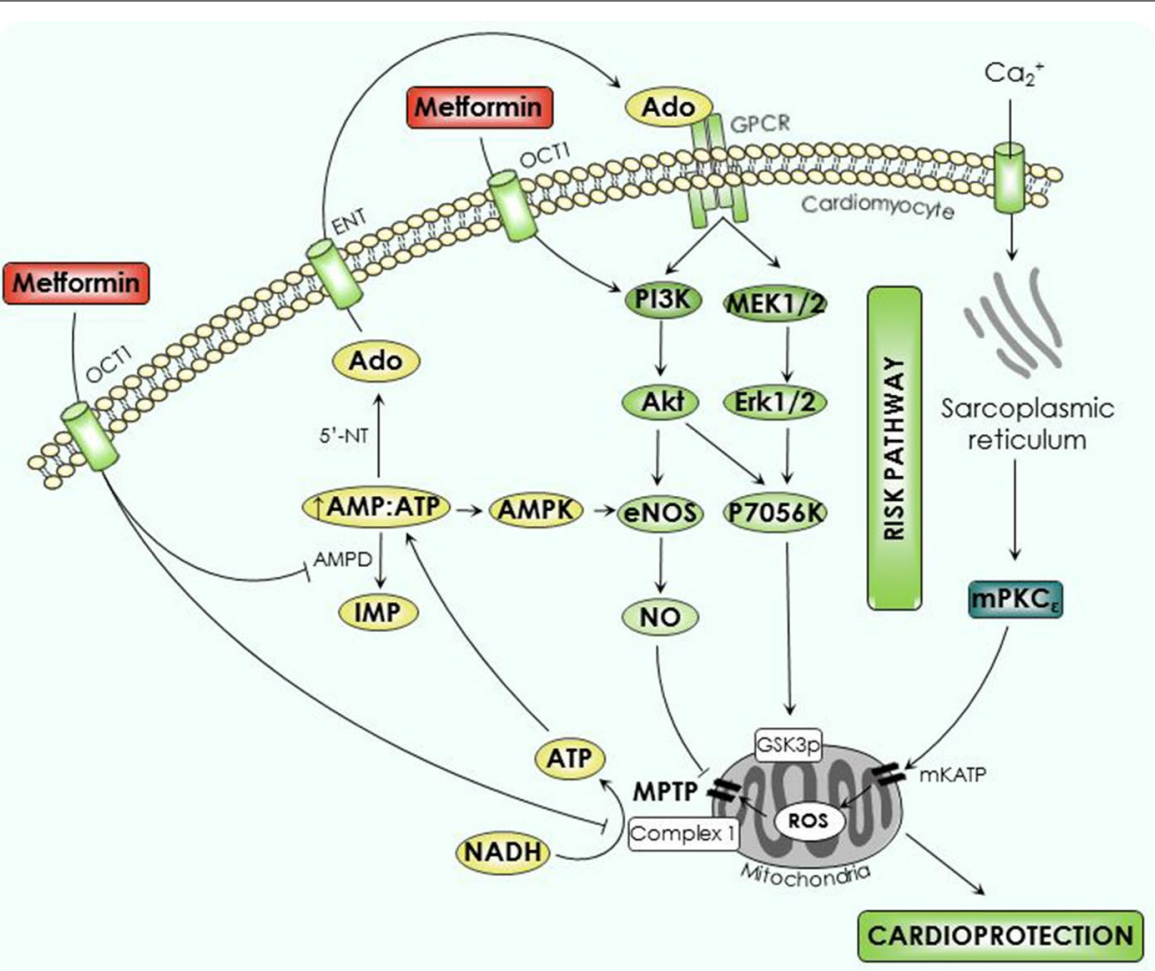

Fig. 1 Proposed cardioprotective mechanisms of metformin. It is suggested that metformin confers cardioprotection by inhibiting mitochondrial complex I and inhibiting AMP deaminase, which both increase cytosolic AMP:ATP ratio. This activates AMPK causing the phosphorylation of eNOS, an integral part of the RISK pathway. Furthermore, increased AMP:ATP facilitates the extracellular diffusion of adenosine and its subsequent activation of the RISK pathway via a G protein-coupled receptor. Metformin may also activate PI3K directly. The RISK pathway inhibits MPTP opening which mitigates the detrimental effects of calcium influx and ROS generation at reperfusion. Ado adenosine, AMP adenosine monophosphate, AMPK adenosine monophosphate-activated protein kinase, ATP adenosine triphosphate, eNOS endothelial nitric oxide synthase, ENT equilibrative nucleoside transporter; Erk, extracellular signal-regulated kinases, GPCR G protein-coupled receptor, IMP inosine monophosphate, MEK1/2 mitogenactivated protein kinase, $m K_{\text {ATP }}$ mitochondrial ATP-sensitive potassium channel, $m P K C$ mitochondrial protein kinase C, MPTP mitochondrial permeability transition pore, NADH nicotinamide adenine dinucleotide, NO nitric oxide, OCT1 organic cation transporter 1, PI3K phosphoinositide 3 kinase, $R I S K$ reperfusion injury salvage kinase, $R O S$ reactive oxygen species. 
despite similar glycaemic control, which has been confirmed elsewhere [23-25]. Despite conflicting evidence from a large meta-analysis of randomized trials, which found no significant effect of metformin on the incidence of cardiovascular events versus active comparator [26], only a handful of studies have specifically evaluated the impact of metformin on IRI after STEMI. For example, Zhao et al. reported that pre-treatment with metformin was associated with a reduction of no-reflow, a phenomenon in which IRI is implicated, in patients with diabetes mellitus after PPCI for STEMI [27]. Importantly, Lexis et al. investigated the effect of pre-treatment with metformin on surrogate markers of AMI size in diabetic patients undergoing PPCI for STEMI in a single centre in the Netherlands [28]. They found metformin treatment to be an independent predictor of smaller MI size, albeit when compared to group on diet alone rather than alternative oral hypoglycaemics. A further study by the same group that administered metformin to patients with STEMI after reperfusion failed to find any improvement in left ventricular function, but here metformin was administered after the critical window of $\mathrm{mPTP}$ opening that is directly associated with IRI [29]. No study has yet evaluated the effect of pre-treatment with metformin on MACE endpoints after PPCI for STEMI.

\section{Missing evidence}

Many of the clinical studies to date have been observational and/or use a placebo/no therapy comparator, which risks confounding by complex and poorly understood metabolic heterogeneity among patients with diabetes, including significant disparity in cardiovascular risk [30]. Furthermore, as mentioned, none have specifically examined the effect on mortality of metformin in the context of IRI, either among patients on metformin therapy at the time of STEMI or by administering metformin intravenously at the time of reperfusion (and therefore mPTP opening). This is likely due to the good availability and widespread uptake of metformin, at least in high-income countries, precluding a control group. Moreover, the potential randomised investigation of metformin in STEMI is hampered by the absence of licensed intravenous preparations and a lack of funding for investigation of a drug that is available off-patent. With this in mind, level 2 evidence may be the best that is realistically possible and as such there is a need for well-designed prospective cohort studies of the potential benefit of periSTEMI metformin. Any such study should be adequately powered to account for the high number of confounding variables inherent in such a study. Finally, it is important to acknowledge the potential deleterious effects of nephrotoxicity and lactic acidosis attendant in the use of metformin in the context of PPCI for STEMI. It is hoped that prospective level 2 studies would clarify the riskbenefit balance of metformin use in this regard, however prior to any prospective randomised study a safety profile for this particular application would be essential.

\section{Conclusions}

Type 2 diabetes and its cardiovascular sequelae represent a major global public health challenge. IRI is a major target for intervention in patients suffering STEMI, and may be of particular benefit in diabetic patients who have inflated cardiovascular mortality compared to non-diabetic patients. There is convincing pre-clinical evidence that metformin, given at the time of IRI, may have a cardioprotective role beyond its glucose-lowering effect. However, despite its availability and safety profile, there is a paucity of clinical studies addressing this hypothesis. To repurpose this potentially beneficial therapy to a global population in need of new treatments for STEMI, especially in regions where PPCI is unavailable, well-designed prospective cohort studies of the potential pleiotropic benefits of metformin on cardiovascular disease, and particularly its benefit in ischaemia-reperfusion injury, are essential.

\section{Abbreviations}

Cl: confidence interval; HR: hazard ratio; IRI: ischaemia-reperfusion injury; STEMI: ST-segment elevation myocardial infarction; PPCl: primary percutaneous coronary intervention.

\section{Authors' contributions}

All authors made substantial contributions to the conception of the article, were involved in drafting the manuscript or revising it critically for important intellectual content, have given final approval of the version to be published, and agree to be accountable for all aspects of the article. All authors read and approved the final manuscript.

\section{Acknowledgements}

Dr Daniel Bromage is an MRC Clinical Research Training Fellow. This work was undertaken at UCLH/UCL who received a proportion of funding from the Department of Health's NIHR Biomedical Research Centres funding scheme of which DM Yellon is a Senior Investigator.

\section{Compliance with ethical guidelines}

\section{Competing interests}

The authors declare that they have no competing interests.

Received: 22 June 2015 Accepted: 3 August 2015

Published online: 14 August 2015

\section{References}

1. World Health Organisation (2014) Global status report on noncommunicable diseases. p xiv.http://apps.who.int/iris/bitstr eam/10665/148114/1/9789241564854_eng.pdf

2. Whiting DR, Guariguata L, Weil C, Shaw J (2011) IDF diabetes atlas: global estimates of the prevalence of diabetes for 2011 and 2030. Diabetes Res Clin Pract 94(3):311-321

3. Morrish NJ, Wang SL, Stevens LK, Fuller JH, Keen H (2001) Mortality and causes of death in the WHO Multinational Study of Vascular Disease in Diabetes. Diabetologia 44(Suppl 2):S14-S21 
4. National Institute for Cardiovascular Outcomes Research (NICOR) (2014) Myocardial Ischaemia National Audit Project Annual Public Report April 2013-March 2014. p 19. https://www.ucl.ac.uk/nicor/audits/minap/ documents/annual_reports/minap-public-report-2014

5. Gibson CM (2004) NRMI and current treatment patterns for ST-elevation myocardial infarction. Am Heart J 148(5 Suppl):S29-S33

6. Keeley EC, Boura JA, Grines CL (2003) Primary angioplasty versus intravenous thrombolytic therapy for acute myocardial infarction: a quantitative review of 23 randomised trials. Lancet 361(9351):13-20

7. Pedersen F, Butrymovich V, Kelbaek H, Wachtell K, Helqvist S, Kastrup J et al (2014) Short- and long-term cause of death in patients treated with primary PCI for STEMI. J Am Coll Cardiol 64(20):2101-2108

8. Turner RC, Millns H, Neil HA, Stratton IM, Manley SE, Matthews DR et al (1998) Risk factors for coronary artery disease in non-insulin dependent diabetes mellitus: United Kingdom Prospective Diabetes Study (UKPDS: 23). BMJ 316(7134):823-828

9. Singh M, Arora R, Kodumuri V, Khosla S, Jawad E (2011) Coronary revascularization in diabetic patients: current state of evidence. Exp Clin Cardiol 16(1):16-22

10. Braunwald E, Kloner RA (1985) Myocardial reperfusion: a double-edged sword? J Clin Invest 76(5):1713-1719

11. Yellon DM, Hausenloy DJ (2007) Myocardial reperfusion injury. N Engl J Med 357(11):1121-1135

12. Piper HM, Garcia-Dorado D, Ovize M (1998) A fresh look at reperfusion injury. Cardiovasc Res 38(2):291-300

13. Staat P, Rioufol G, Piot C, Cottin Y, Cung TT, L'Huillier I et al (2005) Postconditioning the human heart. Circulation 112(14):2143-2148

14. Sivaraman V, Yellon DM (2014) Pharmacologic therapy that simulates conditioning for cardiac ischemic/reperfusion injury. J Cardiovas Pharmacol Therap 19(1):83-96

15. Bhamra GS, Hausenloy DJ, Davidson SM, Carr RD, Paiva M, Wynne AM et al (2008) Metformin protects the ischemic heart by the Akt-mediated inhibition of mitochondrial permeability transition pore opening. Basic Res Cardiol 103(3):274-284

16. Paiva M, Riksen NP, Davidson SM, Hausenloy DJ, Monteiro P, Goncalves L et al (2009) Metformin prevents myocardial reperfusion injury by activating the adenosine receptor. J Cardiovasc Pharmacol 53(5):373-378

17. Calvert JW, Gundewar S, Jha S, Greer JJ, Bestermann WH, Tian R et al (2008) Acute metformin therapy confers cardioprotection against myocardial infarction via AMPK-eNOS-mediated signaling. Diabetes 57(3):696-705

18. Whittington HJ, Hall AR, McLaughlin CP, Hausenloy DJ, Yellon DM, Mocanu MM (2013) Chronic metformin associated cardioprotection against infarction: not just a glucose lowering phenomenon. Cardiovas Drugs Therap Sponsored Int Soc Cardiovas Pharmacotherap 27(1):5-16
19. Paiva MA, Goncalves LM, Providencia LA, Davidson SM, Yellon DM, Mocanu MM (2010) Transitory activation of AMPK at reperfusion protects the ischaemic-reperfused rat myocardium against infarction. Cardiovas Drugs Therap Sponsored Int Soc Cardiovas Pharmacotherap 24(1):25-32

20. Guigas B, Detaille D, Chauvin C, Batandier C, De Oliveira F, Fontaine E et al (2004) Metformin inhibits mitochondrial permeability transition and cell death: a pharmacological in vitro study. Biochem J 382(Pt 3):877-884

21. Detaille D, Guigas B, Chauvin C, Batandier C, Fontaine E, Wiernsperger $\mathrm{N}$ et al (2005) Metformin prevents high-glucose-induced endothelial cell death through a mitochondrial permeability transition-dependent process. Diabetes 54(7):2179-2187

22. Solskov L, Lofgren B, Kristiansen SB, Jessen N, Pold R, Nielsen TT et al (2008) Metformin induces cardioprotection against ischaemia/reperfusion injury in the rat heart 24 hours after administration. Basic Clin Pharmacol Toxicol 103(1):82-87

23. Johnson JA, Majumdar SR, Simpson SH, Toth EL (2002) Decreased mortality associated with the use of metformin compared with sulfonylurea monotherapy in type 2 diabetes. Diabetes Care 25(12):2244-2248

24. Roussel R, Travert F, Pasquet B, Wilson PW, Smith SC Jr, Goto S et al (2010) Metformin use and mortality among patients with diabetes and atherothrombosis. Arch Intern Med 170(21):1892-1899

25. Saenz A, Fernandez-Esteban I, Mataix A, Ausejo Segura M, Roqué i Figuls M, Moher D (2005) Metformin monotherapy for type 2 diabetes mellitus. Cochrane Database Syst Rev (3):CD002966. doi:10.1002/14651858. CD002966.pub3

26. Lamanna C, Monami M, Marchionni N, Mannucci E (2011) Effect of metformin on cardiovascular events and mortality: a meta-analysis of randomized clinical trials. Diabetes Obes Metab 13(3):221-228

27. Zhao JL, Fan CM, Yang YJ, You SJ, Gao X, Zhou Q et al (2013) Chronic pretreatment of metformin is associated with the reduction of the no-reflow phenomenon in patients with diabetes mellitus after primary angioplasty for acute myocardial infarction. Cardiovasc Ther 31(1):60-64

28. Lexis CP, Wieringa WG, Hiemstra B, van Deursen VM, Lipsic E, van der Harst $P$ et al (2014) Chronic metformin treatment is associated with reduced myocardial infarct size in diabetic patients with ST-segment elevation myocardial infarction. Cardiovas Drugs Therapy Sponsored Int Soc Cardiovasc Pharmacotherap 28(2):163-171

29. Lexis CP, van der Horst IC, Lipsic E, Wieringa WG, de Boer RA, van den Heuvel AF et al (2014) Effect of metformin on left ventricular function after acute myocardial infarction in patients without diabetes: the GIPS-III randomized clinical trial. JAMA 311(15):1526-1535

30. Gore MO, McGuire DK, Lingvay I, Rosenstock J (2015) Predicting cardiovascular risk in type 2 diabetes: the heterogeneity challenges. Curr Cardiol Rep 17(7):607

\section{Submit your next manuscript to BioMed Central and take full advantage of:}

- Convenient online submission

- Thorough peer review

- No space constraints or color figure charges

- Immediate publication on acceptance

- Inclusion in PubMed, CAS, Scopus and Google Scholar

- Research which is freely available for redistribution

Submit your manuscript at

www.biomedcentral.com/submit
C Biomed Central 